\title{
High resolution transmission electron microscopy and electronic structure theory investigation of platinum nanoparticles on carbon black
}

Corentin Poidevin, Paul Paciok, Marc Heggen, and Alexander A. Auer

Citation: J. Chem. Phys. 150, 041705 (2019); doi: 10.1063/1.5047666

View online: https://doi.org/10.1063/1.5047666

View Table of Contents: http://aip.scitation.org/toc/jcp/150/4

Published by the American Institute of Physics

\section{Articles you may be interested in}

Multistep and multiscale electron transfer and localization dynamics at a model electrolyte/metal interface The Journal of Chemical Physics 150, 041702 (2019); 10.1063/1.5047033

Face-centered tetragonal (FCT) Fe and Co alloys of $\mathrm{Pt}$ as catalysts for the oxygen reduction reaction (ORR): A DFT study

The Journal of Chemical Physics 150, 041704 (2019); 10.1063/1.5049674

Metal doping in cerium metal-organic frameworks for visible-response water splitting photocatalysts

The Journal of Chemical Physics 150, 041701 (2019); 10.1063/1.5043538

Glycerol electrooxidation on Pd modified Au surfaces in alkaline media: Effect of the deposition method The Journal of Chemical Physics 150, 041703 (2019); 10.1063/1.5048489

Understanding the electrochemical double layer at the hematite/water interface: A first principles molecular dynamics study

The Journal of Chemical Physics 150, 041707 (2019); 10.1063/1.5047930

Elementary kinetics of nitrogen electroreduction on Fe surfaces

The Journal of Chemical Physics 150, 041708 (2019); 10.1063/1.5048036

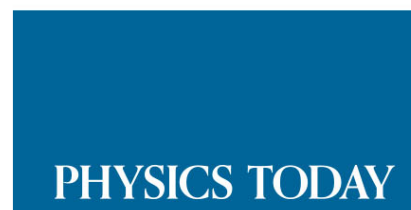

WHITEPAPERS
ADVANCED LIGHT CURE ADHESIVES

Take a closer look at what these environmentally friendly adhesive systems can do

\section{READ NOW}

PRESENTED BY

Q MASTERBOND 


\title{
High resolution transmission electron microscopy and electronic structure theory investigation of platinum nanoparticles on carbon black
}

\author{
Cite as: J. Chem. Phys. 150, 041705 (2019); doi: 10.1063/1.5047666 \\ Submitted: 9 July 2018 - Accepted: 28 September 2018 • \\ Published Online: 26 November 2018
}

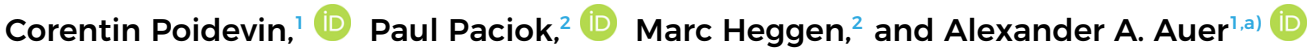 \\ AFFILIATIONS \\ ${ }^{1}$ Max-Planck-Institut für Kohlenforschung, 45470 Mülheim an der Ruhr, Germany \\ ${ }^{2}$ Ernst Ruska-Centre for Microscopy and Spectroscopy With Electrons and Peter Grünberg Institute, \\ Forschungszentrum Jülich $\mathrm{GmbH}, 52425$ Jülich, Germany \\ a) Author to whom correspondence should be addressed: alexander.auer@kofo.mpg.de
}

\begin{abstract}
High Resolution Transmission Electron Microscopy (HR TEM) is used to identify the size, shape, and interface structure of platinum nanoparticles and carbon support of a fuel cell catalyst. Using these insights, models accessible to quantum chemical methods are designed in order to rationalize the observed features. Thus, basal plane and prism face models of the carbon black material are considered, interacting with Pt clusters of sizes up to $1 \mathrm{~nm}$. Particular attention is paid to the electronic structure of the carbon support, namely, the radical character of graphene zig-zag edges. The results show that a stronger interaction is found when the nanoparticle is at the zig-zag edge of a basal plane due to the combination of dispersion interaction with the support structure and covalent interaction with carbon atoms at the edge. In this case, a distortion of both the Pt nanoparticle and the carbon support is observed, which corresponds to the observations from the HR TEM investigation. Furthermore, the analysis of the charge transfer upon interaction and the influence of the potential on the charge states and structure is carried out on our model systems. In all cases, a clear charge transfer is observed from the carbon support to the Pt nanoparticle. Finally, we show that changing the potential not only can change the charge state of the system but can also affect the nature of the interaction between Pt nanoparticles and carbon supports.
\end{abstract}

Published under license by AIP Publishing. https://doi.org/10.1063/1.5047666

\section{INTRODUCTION}

In heterogeneous catalysis, noble metals have been utilized extensively due to their high catalytic activity. However, their low availability and high cost prohibit their use as bulk metals. Hence, to reduce the catalyst loading, supported noble metal nanoparticles (NPs) have been developed and commercially used and it is now possible to control the size, shape, and composition of metal NPs and alloys. ${ }^{1-4}$ In electrochemistry, these catalysts are commonly supported on conductive and porous materials. The choice of the support is far from being anodyne as it has consequences on stability, activity, and cost of the catalyst. ${ }^{5}$ In many applications, carbon based supports are used as they provide crucial support properties such as high surface area and high electric conductivity, and they are easily functionalized and comparatively cheap. A broad diversity of carbon structures with different intrinsic properties is available, such as graphene, carbon black (CB), mesoporous carbon, carbon nanotubes, or carbon nanofibers. ${ }^{6-9}$ However, despite all the qualities of carbon-based materials, their corrosion can become problematic especially under oxidative conditions as often encountered in electrocatalysis. In this respect, carbon supports with high graphitic nature, e.g., carbon nanotubes and carbon nanofibers, are reported to be more stable. ${ }^{5,10}$

One of the main applications of noble metals supported on carbon-based materials in electrocatalysis is in the field of fuel cells and, in particular, in polymer electrolyte membrane fuel cells (PEMFCs). ${ }^{11}$ The most commonly used catalysts for PEMFCs are Pt or Pt-alloy nanoparticles supported on CB. These systems can be put under harsh conditions especially 
during start-up and shut-down where the potential can reach values up to $1.5 \mathrm{~V}$, leading to the degradation of the catalyst. The degradation mechanism is manifold: at these high potentials, the carbon support is thermodynamically driven to be oxidized to $\mathrm{CO}_{2}$ and $\mathrm{CO} ;{ }^{12}$ experimental investigations suggest that dissolution, aggregation, and Oswald ripening are responsible for the degradation of the nanoparticles. In addition to that, detachment of the nanoparticles from the support has also been observed. ${ }^{13,14}$ In an attempt to increase the stability of the catalyst, other carbon supports have been considered like nitrogen-doped carbon black ${ }^{15}$ or Hollow Graphitic Spheres (HGSs). ${ }^{16}$

Among many aspects that have to be considered, the nature and the strength of the interaction between the metal NP and the support plays an important role for the catalytic efficiency, the catalyst loss, and the charge transfer properties. Thus, understanding the interaction between these metal clusters and the different carbon-based supports is paramount. Yet, due to the size, the irregular shapes, and the large difference in the chemical nature of the components of these materials, only little information can be collected on the interaction between the two moieties. Nonetheless, microscopy technics like high-resolution transmission electron microscopy (HR TEM) are able to provide crucial information on the size and shape of the NPs as well as their approximate positions on the support. It can also give insight into the support structure and particularly on its graphitic content. Subsequently, these valuable data can be used to build smaller models accessible to atomistic simulations in order to approach features that cannot be probed otherwise.

Thus, theoretical work aiming to understand the basics of the interaction between Pt clusters and graphitic supports has been the focus of many studies in the last 15 years. ${ }^{17-29}$ However, from a computational point of view, modeling such systems remains very challenging. Even when considering a very small portion of the actual electrocatalyst, still a few hundred atoms should be included in the model. Therefore, the vast majority of the studies were performed using density functional theory (DFT) with a plane-wave basis and periodic boundary conditions to reduce the number of carbon atoms and to mimic the properties of an extended graphene sheet support. Additionally, most of the early work used small metal clusters, up to 13 platinum atoms, as NP models. In this context, following the work of Xiao and Wang on the relative stability of planar and three-dimensional Pt clusters in vacuum, ${ }^{17}$ Okazaki-Maeda et al. ${ }^{18}$ showed that for clusters with less than 10 atoms, planar and three-dimensional structures were close in energy on a graphene support and that for larger clusters, the three-dimensional structures were unambiguously more stable than the planar ones. More recently, Verga et al. ${ }^{19}$ were able to perform a study on clusters of up to $309 \mathrm{Pt}$ atoms and confirmed and extended what Okazaki-Maeda et al. had found. Beside these structural changes, it was found that the size of the NPs also influences the nature of their interaction with graphene basal planes.
Indeed, with the availability of correction schemes for dispersion interactions, ${ }^{30,31}$ in one of our previous studies, ${ }^{21}$ we showed that there is a transition between an interaction with covalent character for smaller clusters and a dispersiondominated interaction for larger particles on the graphene basal plane. The same observations were made in the studies of Ramos-Sanchez and Balbuena ${ }^{22}$ and Verga et al. ${ }^{19}$ These conclusions are in agreement with experimental observations of rather mobile nanoparticles on graphene basal planes, ${ }^{32}$ as dispersion interactions are inherently non-directional.

In addition to pristine graphene basal planes, defective basal planes have also been studied. Several studies on $\mathrm{Pt}_{13}$ report that the presence of defects on a basal plane, such as a carbon vacancy and 5- or 7-membered rings, increases the interaction between the metal cluster and the carbon support. ${ }^{26,27,29}$ In our previous work, defects such as 5- or 7membered rings and the substitution of a carbon atom by a heteroatom (namely, N, O, and $\mathrm{S}$ ) on a basal plane were also considered in interaction with a $\mathrm{Pt}_{37}$ cluster. The results showed that these defects increase the covalent contribution to the interaction but can also increase the average distance between the metal cluster and the support. This decreases the dispersion interaction, thus resulting in an equal or lower interaction energy compared to that on a pristine basal plane.

As already mentioned, most of the theoretical studies on the interaction between Pt NPs and carbon supports were done using extended graphene sheets. However, it is clear that the structure of most carbon-based supports is far from the ideal extended graphene basal plane. Yet these models reveal the predominance of dispersion interactions for large NPs in contact with graphitic structures, thus explaining their mobility on these surfaces. Additionally, an experimental study on few layer graphene using TEM showed that Pt nanoparticles were preferentially located at topographical defects such as steps and edges after deposition. ${ }^{33}$ On this basis, in order to broaden our understanding of the interactions and degradation mechanisms present in the real catalyst material, topographical defects have to be considered in the design of the support models. Moreover, CB is still the most commonly used carbon support for PEMFCs. Hence, trying to build a model based on its structure would be of great interest.

In the literature, carbon black is described as spherical particles of 10-50 nm diameter. These particles are composed of stacks of polycyclic aromatic hydrocarbons (PAHs). ${ }^{34}$ While the macroscopic characteristics of this material are well known, little is known about the precise size and shape of these PAHs and the interaction sites with metallic nanoparticles. As mentioned above, TEM can be a powerful tool to unravel specific structural aspects of these materials. Thus, we will use TEM images to define relevant carbon model structures. From the analysis of the TEM images, we will discuss and justify the use of PAHs in a given size range instead of extended graphene sheets as carbon support models for the description of this type of electrocatalyst. An 
evaluation of the sizes and potential locations of the Pt nanoparticles will also be done to further improve the computational model.

The fact that $\mathrm{CB}$ and most carbon-based supports are composed of PAHs or at least non-extended graphene sheets demonstrates the relevance of understanding their specific properties. It is now well known that the electronic ground state of PAHs can have radical character. ${ }^{35,36}$ This was first considered to explain the instability of large polyacenes and nanoribbons. ${ }^{37}$ Theoretical studies showed that, depending on their size and shape, PAHs could exhibit a wide range of electronic spin states (closed or open shell singlet, triplet. . .). ${ }^{38-43}$ In recent years, large polyacenes and nanoribbon analogs were synthetized and studied, confirming the presence of a radical ground state in these systems. ${ }^{35,37,44}$ In this study, we will discuss the influence of the shape of the carbon support on its electronic ground state and its impact on the interaction with $\mathrm{Pt}$ nanoparticles. The charge transfer occurring upon interaction will also be examined and compared to trends reported in the literature. Furthermore, as these are molecular properties, all our calculations will be carried out using a cluster approach rather than periodic boundary conditions.

In the last part of this work, we will address a feature specific to electrochemistry: the presence of an applied electrode potential, for convenience referred to as potential in the following. When modeling electrocatalytic systems, the potential is often only taken into account in the cases of faradic reactions. Yet changing the potential can result in the change of the charge state of the system, i.e., its reduction (low potentials) or its oxidation (high potentials), which in turn can affect its stability and its change transfer properties. Computationally, a constant potential can be included either by directly converging the system at a given potential (using a fractional charge SCF scheme) $)^{45}$ or by using an interpolation scheme between integer charge states. ${ }^{46}$ In the present work, we will only consider potential domains at which the different charge states of our "molecular" systems are calculated to be stable. Thus, we will start by looking at its influence on one of our support models and a Pt cluster separately and then on the interacting system.

\section{A. Computational details and theory}

The calculations were carried out using the ORCA 4.0 program package. ${ }^{47}$ The B3LYP ${ }^{48,49}$ density functional with the RIJCOSX ${ }^{50}$ approximation and the D3 dispersion correction $^{31,51}$ was used with the def2-SVP and def2-TZVP52 basis sets and def $2 / \mathrm{J}$ auxiliary basis set. ${ }^{53}$ For platinum atoms, an effective core potential for 60 electrons was used. ${ }^{54}$ The geometries of the carbon support models and the Pt nanoparticles were separately optimized at the RIJCOSX-B3LYPD3/def2-SVP level of theory. Note that a spin search was performed on each platinum nanoparticle. Subsequently, the Pt clusters were placed over the supports and the geometries were optimized at the same level of theory. From these geometries, single-point calculations were carried out at the RIJCOSX-B3LYP-D3/def2-TZVP level of theory.
The interaction energies $E_{\text {int }}$ were calculated as the

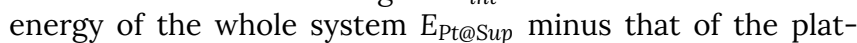
inum nanoparticle $E_{P t}$ and the support $E_{S u p}$ at their respective optimum geometries,

$$
E_{i n t}=E_{P t @ S u p}-E_{P t}-E_{\text {Sup }} .
$$

In order to discuss the influence of the potential on our systems, we will use the following definitions of the electron chemical potential, $\mu$, and of the reduction potential, $\mu^{\circ}$. By definition, the electron chemical potential $\mu$ of a given system is the derivative of the energy with respect to the number of electrons [Eq. (2)]. Hence it can be approximated by calculating E for various charge states Q [Eq. (3)],

$$
\begin{aligned}
\mu & =\frac{\delta \mathrm{E}}{\delta \mathrm{N}}, \\
\mu^{\mathrm{Q}} & =\frac{\mathrm{E}^{\mathrm{Q}-1}-\mathrm{E}^{\mathrm{Q}+1}}{2} .
\end{aligned}
$$

The reduction potential $\mu^{\circ}$ of a couple $\mathrm{M}^{\mathrm{Q}+1} / \mathrm{M}^{\mathrm{Q}}$ can be approximated by the electronic energy difference $E\left[M^{\mathrm{Q}}\right]-E\left[\mathrm{M}^{\mathrm{Q}+1}\right]$. In a molecular picture, a system $\mathrm{M}$ will be in a charge state $\mathrm{Q}$ for values of potential between $\mu^{\circ}\left[\mathrm{M}^{\mathrm{Q}} / \mathrm{M}^{\mathrm{Q}-1}\right]$ and $\mu^{\circ}\left[\mathrm{M}^{\mathrm{Q}+1} / \mathrm{M}^{\mathrm{Q}}\right]$. The electron chemical potentials and the reduction potentials thus obtained can be converted to a difference of potential $U$ versus the standard hydrogen electrode (SHE) by following the ansatz originated by Trasatti [Eqs. (4) and (5)], ${ }^{55}$

$$
\begin{aligned}
\mathrm{U}(\mathrm{V} v \mathrm{sSHE}) & =-\mu^{\mathrm{Q}}-4.44(\mathrm{eV}), \\
\mathrm{E}^{\circ}(\mathrm{V} v \mathrm{sSHE}) & =-\mu^{\circ}-4.44(\mathrm{eV}) .
\end{aligned}
$$

\section{B. Experimental details}

For the analysis of the platinum and carbon structure, the chromatically corrected high resolution transmission electron microscope Titan 50-300 PICO (FEI Company) was used at $80 \mathrm{kV}$. To achieve a strong image contrast, the negativespherical-aberration imaging (NCSI) technique was applied. ${ }^{56}$ The images were acquired with a Gatan OneView camera. For the sample preparation, a gold TEM finder grid (S147A9, Plano $\mathrm{GmbH}$ ) was drop casted with a highly diluted dispersion (ultrapure water, $18.2 \mathrm{M}, 4 \mathrm{ppm}$ TOC, Millipore) of the catalyst HiSPEC 2000 (Johnson Matthey) and dried in air. The platinum particle size distribution was determined manually from at least 200 individual particles with the software Image J.

\section{HR TEM IMAGES OF PLATINUM NANOPARTICLES SUPPORTED ON CARBON BLACK}

As a reference catalyst, Johnson Matthey's HiSPEC 2000 catalyst was chosen. Here, the active material platinum is supported on the widely used carbon black Vulcan XC 72R. Due to the low loading of $10 \mathrm{wt}$. \% platinum, the probability of overlapping platinum particles is reduced and the view on the platinum carbon interface is not obscured. 
Figures 1(a) and 1(d) show representative HR TEM images of Johnson Matthey's fuel cell catalyst Hispec 2000. Platinum particles with an average size of $2.1 \mathrm{~nm} \pm 0.6 \mathrm{~nm}$ are supported on the commercial carbon black Vulcan XC 72R. From Figs. 1(d) and 1(g), the structure of the carbon black can be deducted as onion-like. The analysis of the different shells of those onion structures reveals contrast variations within the shells. The onion shells consist of smaller segments which can be attributed to PAH. The observed length of these PAHs shows huge fluctuations with ranges from sub nm up to several $\mathrm{nm}$. The distance between the various layers corresponds to the graphite (002) d-spacing of $3.34 \AA$.

Most of the platinum particles show the presence of surface facets (Fig. 1). The particle in Fig. 1(b), which is oriented with the beam direction along its [110] direction, shows $\{111\}$ and $\{001\}$ facets which are characteristic for a cuboctahedral or truncated-octahedral shape. At the interface between the particles and the carbon support, however, distortions and/or deviation from the well-faceted shape are observed [see Figs. 1(b), 1(c), and 1(e)]. The platinum particles are flattened, and the platinum atoms in contact with the PAHs mimic the shape and curvature of the carbon structure leading to a zig zag shape of the platinum interface. Also, the analysis of the $\mathrm{Pt} / \mathrm{C}$ interface of various particles indicates a preference of the platinum (111) planes as the contact sites. The hexagonal carbon network close to the (001) graphite plane has a periodicity of $2.84 \AA$. Due to the similarity of the graphite periodicity and the Pt-Pt distance in the (111) plane (2.77 Angstrom), there might be a preference for epitaxial growth between the Pt (111) planes and (001) graphite planes as Willinger et al. pointed out. ${ }^{57}$ It is noteworthy that besides a distortion of the platinum contact area, perturbations of the graphite PAH layers next to platinum particles are present (see red arrows in Fig. 1). An investigation of the PAHs "covered" by platinum [see Fig. 1(b)] shows an increase of the curvature of the graphite layers compared to graphite layers without platinum in the vicinity. This might indicate a direct or indirect effect of the platinum particles on the graphite structure and its electron density-or, vice versa, the necessity of defects to be present for the formation of platinum particles at a given site on the support.

\section{THE COMPUTATIONAL MODELS}

For computational feasibility reasons, it is obviously not possible to calculate an extended region of a Pt cluster at the carbon system, as depicted in Fig. 1. One therefore needs to create models that include most of the essential observed features of the experimental system and thus have access to properties that cannot be probed, such as interaction energies and mechanisms.

\section{A. Pt nanoparticles}

The HR TEM images of Pt NPs supported on CB reveal the presence of cuboctahedral or truncated-octahedral shaped NPs with the Pt(111) face as the preferential interaction surface. In one of our previous studies, ${ }^{21}$ platinum clusters containing 10, 18, and 37 atoms were used as catalyst models. The latter, of hemispherical cuboctahedral shape, has a hexagonal bottom plane (111 face), thus reproducing the main shape features of the observed NPs. Its size is of about $1 \mathrm{~nm}$, which is slightly smaller than the experimental NPs. However, our previous study showed that this size was sufficient to reproduce the non-directional interaction (dominated by dispersion) with graphene basal planes explaining the mobility of larger Pt NPs on this surface. It will hence be used as the Pt NP model of larger NPs in this study. The other two clusters will also be considered to evaluate the behavior of smaller NPs on the interaction with support models (see Fig. 2).

\section{B. The carbon models}

According to the above HR TEM images of Pt NPs on carbon black, a wide range of local structures can be accessible to a nanoparticle. The contact seems to be made either on a PAH basal plane, at its edges, or, to a lesser extent, on a prism face of a stack. For each of the previous cases, different topologies (zig-zag vs armchair edge) and defects (heteroatoms and functional groups) might be found, leading to a broad spectrum of possible interaction sites. In this study, we will use two PAHs of different shapes as carbon models for NPs interacting with a basal plane. We will then consider two stackings of PAHs as a model for prism faces. First, we will start

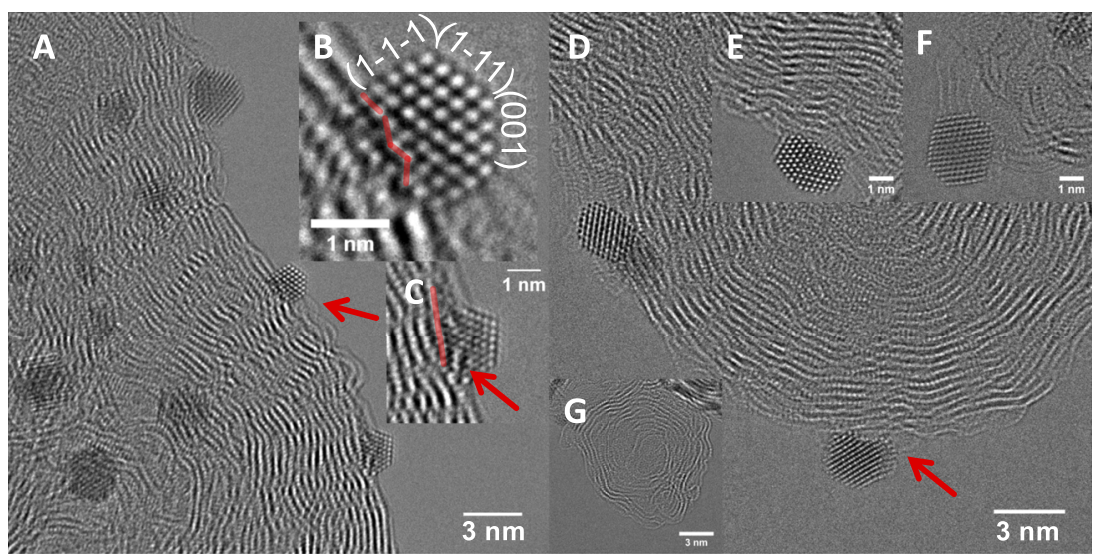

FIG. 1. [(a) and (d)] Representative HR TEM of platinum particles supported by Vulcan XC 72R (scale bar $3 \mathrm{~nm}$ ). (b), (c), (e), and (f) show the interface between the carbon support and the platinum particles. (g) Onion-like structure of a carbon black support particle. 

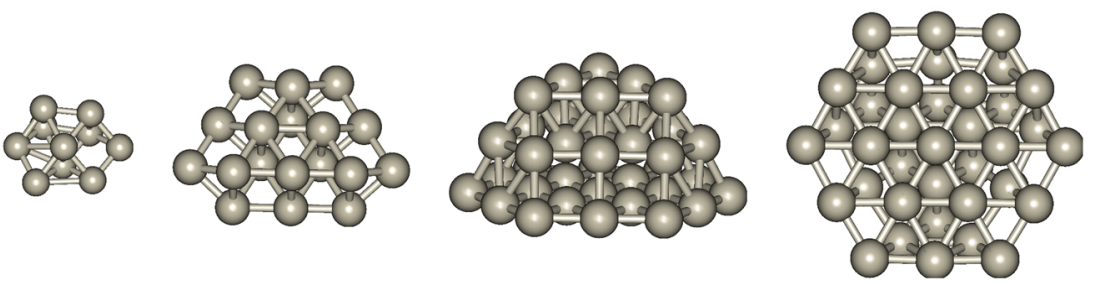

FIG. 2. From left to right: $\mathrm{Pt}_{10}, \mathrm{Pt}_{18}$, and side and bottom (111 surface) view of $\mathrm{Pt}_{37}$ clusters. with some general considerations on the electronic ground state of PAHs.

\section{On the electronic structure of PAHs}

From the information obtained from the HR TEM images, the carbon support model can be built from one or several PAHs in the size range of 1 to a few nm (i.e., 4 to more than 8 benzene rings). Thus, the construction of a basal plane model can be seen as a fairly simple 2D problem with only two parameters, the shape and the width of the sheet(s). However, the nature and size of its edges can have a great impact on the electronic structure of the model. For instance, the two families of linear PAHs, which are the acenes (with zigzag edges) and the phenacenes (with armchair edges), exhibit very different electronic properties as the number of rings increases. Plasser et al. showed, using the multireference averaged quadratic coupled-cluster (MR-AQCC), that on the one hand, acenes with more than 5 rings have a radical character, i.e., open shell singlet ground state, which increases with the length of the system, ${ }^{38}$ consistent with pentacene being the largest well-characterized acene. ${ }^{58}$ On the other hand, no radical character was found in the phenacene series they studied (up to 10 rings). They also studied the influence of the size and the shape of two-dimensional PAH families, the periacenes and the circumacenes. In both cases, the radical character increased with the size of the systems, but despite their resemblance, different degrees of radical character were obtained.

To make sure our level of theory was appropriate to describe this specific feature of PAHs, we investigated two isomeric series: $5 \mathrm{zNa}$ and $\mathrm{Nz} 5 \mathrm{a}$ (see Fig. 3), with $\mathrm{Nz}$ and $\mathrm{Na}$ being the number of benzene rings at the zig-zag edge and armchair edge, respectively, with $\mathrm{N}$ going from 1 to 5 . As in the study of Plasser et al., our results suggest an increase of the radical character with the size of the system. Another interesting observation is that in these series, PAHs with longer armchair edges than zigzag ones are calculated to be both more stable and have no or a smaller radical character than their isomer, as shown in Fig. 3. A common trend in PAHs is that the unpaired electrons are always localized at the zig-zag edges. Thus, one can expect a large difference in reactivity between zigzag and armchair edges.
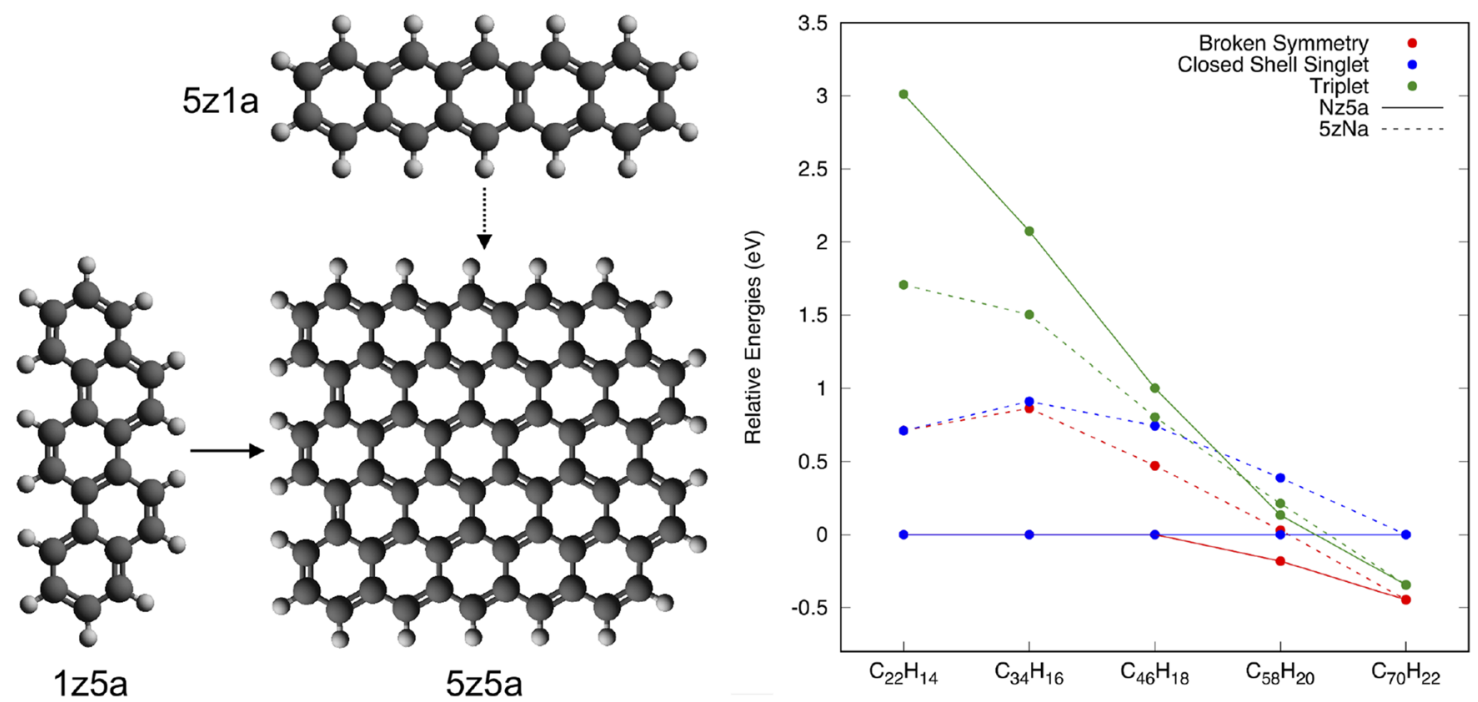

FIG. 3. Left: $5 z \mathrm{Na}$ and Nz5a structures. Right: Relative closed shell singlet and triplet end broken symmetry energies of Nz5a and $5 z N a$ PAHs with respect to that of Nz5a closed shell singlet states (B3LYP/def2-TZVP). 


\section{Basal plane models}

To study the influence of the radical character, i.e., the closed or open shell singlet ground state of PAHs on the interaction with Pt nanoparticles at the basal plane, we used the following models: (i) a D6h symmetry one, $\mathrm{C}_{96} \mathrm{H}_{24}$ (B1), calculated to be a close shell singlet ground state and (ii) a $\mathrm{C} 2 \mathrm{v}$ symmetry one, $\mathrm{C}_{126} \mathrm{H}_{30}$ (B2), calculated to be an open shell singlet ground state (see Fig. 4). Both supports have about the same "length" and "width" and are in the size range of the observed PAHs in carbon black, $\sim 1.7 \mathrm{~nm}$ and $1.6 \mathrm{~nm}$, respectively. From the difference in energy with the close shell singlet, $0.76 \mathrm{eV}$, and the $\left\langle\mathrm{S}^{2}\right\rangle$ value, 1.82 , of the broken symmetry solution (see Table I), it is clear that $\mathrm{B} 2$ has a radical character. To estimate the number of unpaired electrons, one can use the natural orbital occupations, $n$, and the formalism of Ref. 59 with the recommended formula $n_{i}^{2}\left(2-n_{i}\right)^{2}$. Using this method, the calculated number of unpaired electrons in B2 is about 2.6. Also, as shown in Fig. 4, the unpaired electrons are localized at the zig-zag edges of the support. Furthermore, in order to assess their potential relative abundance in the CB material, one needs to estimate their relative stability. For this purpose, the energy per carbon of the complete oxidation $\left(\mathrm{C}_{n} \mathrm{H}_{m}+(\mathrm{n}+\right.$ $\mathrm{m} / 4) \mathrm{O}_{2} \rightarrow \mathrm{nCO}_{2}+\mathrm{m} / 2 \mathrm{H}_{2} \mathrm{O}$ ) was computed for both systems, along with that of smaller PAHs of the same symmetries (see Fig. S1 of the supplementary material). The results reveal that the support models B1 and B2 are fairly close in energy per $\mathrm{C}$ atom and can thus be viewed as equally thermodynamically stable.

In graphitic carbon materials, these PAHs would interact with the rest of the carbon support through stacking and covalent interactions at their edges. Therefore, some of the carbon atoms were kept fixed during the geometry optimization process, while allowing the atoms in the vicinity of the nanoparticle to relax (see pink carbon atoms in Fig. 4).

\section{Prism face models}

Contrary to the 2D basal plane models, the 3D nature of stacked prism faces makes its modeling substantially more complex. The topology of the PAH edge can either be zigzag or armchair, which has, again, direct consequences on the electronic structure in its vicinity, and it also induces different geometric constraints for heteroatoms and chemical groups. As the nanoparticle is to be in contact with several PAHs, one
TABLE I. Relative energies, in $\mathrm{eV}$, of the first triplet $(\mathrm{T})$ and the broken symmetry state (BS) with respect to the close shell singlet (CSS) state of the two basal plane models calculated at the B3LYP/def2-TZVP level.

\begin{tabular}{lccccc}
\hline \hline & \multicolumn{2}{c}{$\mathrm{B} 1$} & \multicolumn{2}{c}{$\mathrm{B} 2$} \\
\cline { 2 - 5 } & $\mathrm{E}(\mathrm{eV})$ & $\left\langle\mathrm{S}^{2}\right\rangle$ & & $\mathrm{E}(\mathrm{eV})$ & $\left\langle\mathrm{S}^{2}\right\rangle$ \\
\hline $\mathrm{CSS}$ & 0.00 & 0.00 & 0.00 & 0.00 \\
$\mathrm{~T}$ & 1.45 & 2.03 & -0.60 & 2.13 \\
$\mathrm{BS}$ & $\ldots$ & $\cdots$ & -0.76 & 1.82 \\
\hline \hline
\end{tabular}

has to choose which stacking to use. The most stable one known for graphene is the hexagonal ABA stacking with an ideal interlayer distance of $3.34 \AA$. However, the low interlayer binding energy allows for a broad distribution of stacking variants and orientation angles. Also, variations in the interlayer distance from 2.90 to $4.00 \AA$ can be observed in highly defective systems.

Due to the computational cost of calculating several large PAHs in interaction, the number of sheets and the size of each individual layer have to be minimized while having a total edge area larger than the radius of the platinum NPs we are considering (up to $1 \mathrm{~nm}$ ). In this work, we choose to focus on the zig-zag edge prism face. In this context, the smallest, yet pertinent with respect to the HR TEM images, model of prism face would have to have the same shape as the bottom surface of the hemispherical cuboctahedral nanoparticle (see Fig. 2). Choosing sheets of 7, 6, and 5 rings assembled in a zig-zag edge fashion lead to the desired prism face model with the widths of about 1.7, 1.5, and $1.2 \mathrm{~nm}$, respectively.

The thickness of the stack, or the number of rows of 6-membered rings of each sheet, also has to be carefully considered. Several constraints have to be taken into account:

(i) the electronic structure of each model sheet has to resemble that of a bigger, more realistic one, i.e., in our case of zig-zag edges, having an open shell character in the ground state;

(ii) during the geometry optimization of the whole system (support plus nanoparticle), the position of the bottom atoms of the sheets has to remain fixed to mimic the effect of the stacking of a bigger system, thus taking
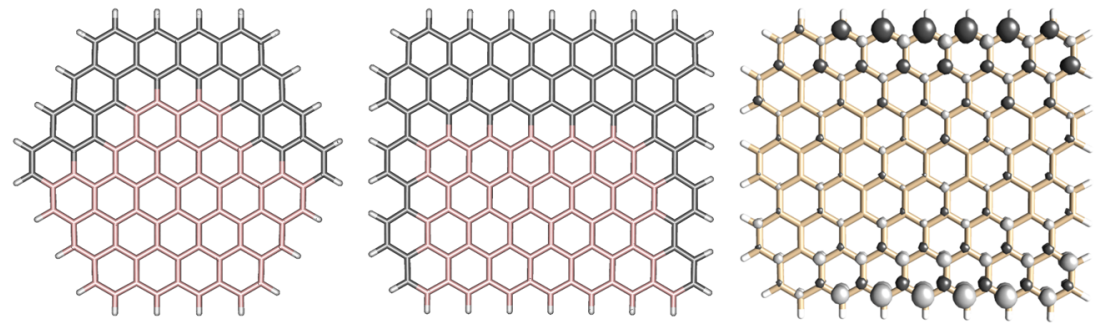

FIG. 4. Left: B1 (D6h symmetry), middle: B2 (C2v symmetry), and right: the spin density of the broken symmetry solution of B2 (isovalue of \pm 0.005 ). Highlighted carbons correspond to the non-constrained ones. 
TABLE II. Relative energies, in $\mathrm{eV}$, of the first triplet (T) and the broken symmetry state (BS) with respect to the close shell singlet (CSS) state of the three different units composing the prism face model calculated at the B3LYP/def2-TZVP level.

\begin{tabular}{|c|c|c|c|c|c|c|}
\hline & \multicolumn{2}{|c|}{$\mathrm{C}_{34} \mathrm{H}_{16}$} & \multicolumn{2}{|c|}{$\mathrm{C}_{40} \mathrm{H}_{18}$} & \multicolumn{2}{|c|}{$\mathrm{C}_{46} \mathrm{H}_{20}$} \\
\hline & $\mathrm{E}(\mathrm{eV})$ & $\left\langle\mathrm{S}^{2}\right\rangle$ & $\mathrm{E}(\mathrm{eV})$ & $\left\langle\mathrm{S}^{2}\right\rangle$ & $\mathrm{E}(\mathrm{eV})$ & $\left\langle\mathrm{S}^{2}\right\rangle$ \\
\hline CSS & 0.00 & 0.00 & 0.00 & 0.00 & 0.00 & 0.00 \\
\hline $\mathrm{T}$ & 0.50 & 2.05 & 0.20 & 2.06 & -0.02 & 2.07 \\
\hline BS & -0.02 & 0.39 & -0.11 & 0.82 & -0.25 & 1.11 \\
\hline
\end{tabular}

only one row would not allow enough flexibility to the top atoms;

(iii) the high computational cost of optimizing the geometry of such a big system is an important limitation to the number of rows that can be used.

After consideration of these three points, the use of two rows of 6-membered rings for each sheet appeared to be the best compromise. Indeed, all three of them show an open shell singlet ground state character (see Table II). Constraining the position of the bottom carbon atoms still allows for some flexibility for the middle and top ones, and with 282 atoms in total for the support plus at most 37 platinum atoms, this model system is still in the range of what can be calculated using a cluster approach.

A fixed distance between the sheets has to be imposed due to the constraints on the bottom atoms of each sheet. As mentioned above, the geometry optimizations are carried out at the B3LYP-D3/def2-SVP level followed by a single point calculation at the B3LYP-D3/def2-TZVP level. We optimized the $\mathrm{C}_{34} \mathrm{H}_{16}$ dimer at these two levels and obtained interplane distances of 3.29 and $3.39 \AA$, respectively. These values are close to the ideal interlayer stacking of graphene, $3.34 \AA$, the value which we ultimately chose to use as interplane distance for our models. Finally, we used the ABA stacking in two different arrangements for our models of the graphite prism face, one in a planar prism face arrangement (P1) and the other in a wavy prism face arrangement (P2; see Fig. 5). Thus, each sheet

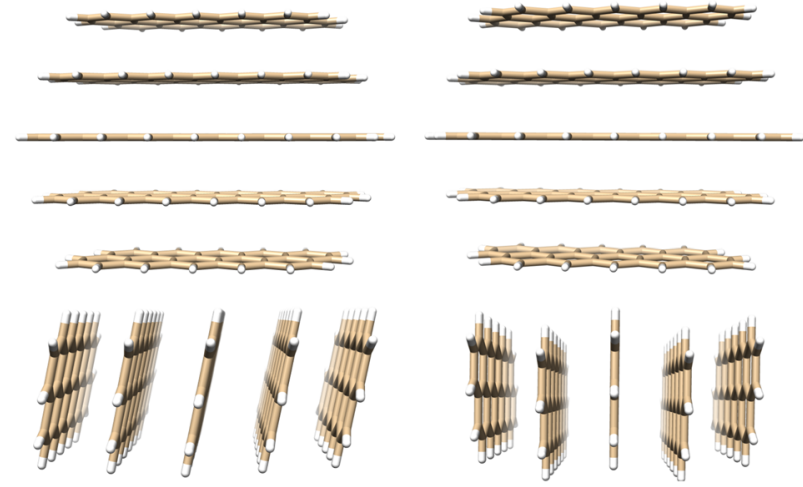

FIG. 5. The two prism face support models. Left: in a planar prism face arrangement $\mathrm{P} 1$; right: in a wavy prism face arrangement $\mathrm{P} 2$. was individually optimized and placed $3.34 \AA$ from each other in an ABA stacking.

\section{RESULTS AND DISCUSSION}

In Sec. IV A, we will start by looking at the structural differences between Pt NPs interacting with a closed shell basal plane and an open shell one. We will then discuss the implications on the interaction energies and natures.

\section{A. Pt nanoparticles at the graphene basal plane}

Considering the $\mathrm{Pt}_{37}$ at $\mathrm{B} 1$, whether the geometry optimization is done with all the carbon atoms fixed or allowing some to relax (as shown in Fig. 4), the NP remains close to the center of the support at a minimum distance of $2.9 \AA$. On the other hand, when the NP is on top of the B2 support, in both cases (fixed or partially relaxed carbons), it migrates to one of the zig-zag edges of the PAH (see Fig. 6). A deviation from the planarity of the $\mathrm{sp}^{2}$ hybridization towards the tetrahedral $\mathrm{sp}^{3}$ hybridization is observed on two of the carbons from this edge. Their distances to the closest Pt atom are only $2.2 \AA$, which is much shorter than in the previous case. This indicates the formation of covalent bonds between the $\mathrm{Pt}_{37}$ nanoparticle and the sheet. Thus, upon interaction of a Pt NP with a radical zig-zag edge, a distortion of both the NP and the $\mathrm{PAH}$ is observed which corresponds to the observations of distortions between NPs and PAHs in the HR TEM investigation [Fig. 1(b)].

To evaluate the influence of the particle size and the presence of defects on larger particles on the nature of the interaction, a $\mathrm{Pt}_{18}$ cluster and a $\mathrm{Pt}_{10}$ cluster were also studied. $\mathrm{Pt}_{18}$ and $\mathrm{Pt}_{10}$ do not have this well-defined bottom surface, and the coordination number of the platinum atoms at the interface is smaller. As already discussed in our previous study, the smaller the NP, the more covalently it interacts with a closed shell graphene basal plane, as can be seen in the structures of $\mathrm{Pt}_{10}$
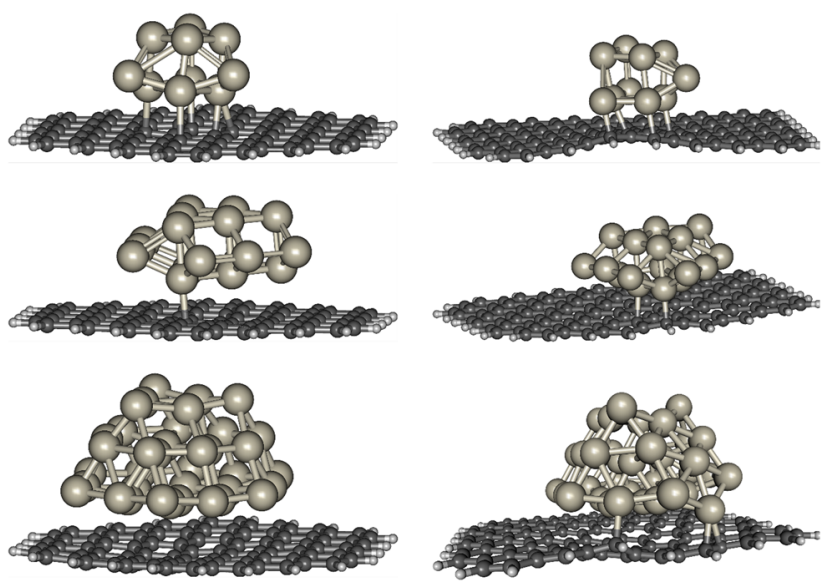

FIG. 6. Optimized structures of $\mathrm{Pt}_{10}, \mathrm{Pt}_{18}$, and $\mathrm{Pt}_{37}$ interacting with $\mathrm{B} 1$ (left) and B2 (right). 
and $\mathrm{Pt}_{18}$ interacting with the closed shell $\mathrm{B} 1$ support. Indeed, the shortest distances between Pt atoms and the support lie between 2.4 and $2.6 \AA$ for the $\mathrm{Pt}_{18}$ cluster and decrease from 2.3 to $2.5 \AA$ for the $\mathrm{Pt}_{10}$ cluster. In the case of the open shell support (B2), as for the larger NP, the minimum energy structure is found when the NP is at one of the zig-zag edges of the $\mathrm{PAH}$. The shortest distance between Pt atoms and the support is now $2.2 \AA$ for the $\mathrm{Pt}_{18} \mathrm{NP}$ and $2.1 \AA$ for the $\mathrm{Pt}_{10} \mathrm{NP}$, indicating stronger covalent interactions with this support, as in the case of the larger $\mathrm{Pt}_{37} \mathrm{NP}$.

The calculated interaction energies as well as their decomposition in terms of dispersive and covalent character are displayed in Fig. 7 (values can be found in Table S1). One can see that for these two supports, the overall interaction increases with the size of the NP. Another common trend observed as the particles get larger is the increase of the dispersion contribution to the interaction and the decrease of its covalent counterpart. The former can be attributed to the increase of the number of platinum atoms in contact with the support while the latter can be attributed to the increase of the coordination number of these atoms. As expected from the analysis of the optimized structures, an increase in the covalent contribution to the interaction is observed when going from the closed shell support to the open shell one while its dispersive counterpart remains fairly constant. Thus, the interaction of Pt NPs is calculated to be stronger at the zig-zag edges of an open shell support than on a basal plane. Additionally, for large particles, the interaction is non-directional on the basal plane, while that at the zig-zag edges involves covalent bonds and so provides an anchor to the NPs. This can explain why after deposition Pt NPs were observed at graphene edges rather than on basal planes. ${ }^{33}$

\section{B. Pt nanoparticles at the graphite prism face}

The optimized geometry of the $\mathrm{Pt}_{37}$ cluster on $\mathrm{P} 1$ shows very little displacement of the non-constrained carbon and hydrogen atoms on the support and no significant change in the $\mathrm{C}-\mathrm{H}$ bond lengths due to the presence of the $\mathrm{Pt}$ nanoparticle (see Fig. 8). The interaction energy is calculated to be very close to the one obtained for the interaction of $\mathrm{Pt}_{37}$ on $\mathrm{B} 1$ (see Fig. 7). However, from the decomposition of the interaction energy, one can see that the nature of the interaction is not exactly the same. Indeed, the dispersion contribution has decreased due to the fact that in this case, the flat lower surface of the NP is on top of 13 hydrogen atoms at $\sim 2.0 \AA$ and 13 carbon atoms at $\sim 3.0 \AA$, whereas it was directly on top of 33 carbon atoms at $\sim 2.9 \AA$ on B1. Going from this planar prism face to that of the P2 support, only a small deformation of the lower $\mathrm{Pt}_{37}$ (111) face is observed, resulting in higher Pt-support distances, thus lower dispersion interaction.

For the $\mathrm{Pt}_{18}$ cluster, as can be seen in Fig. 7, the top of the sheets below the NP is slightly bent. More interestingly, the hydrogens below the platinum atoms moved off the plane of the sheet to make room for the $\mathrm{Pt}-\mathrm{C}$ interactions. Indeed, while the $\mathrm{Pt}-\mathrm{H}$ distances remain the same as in the previous cases, the minimum Pt-C distances are now about 2.6 ̊. This is reflected in the interaction energy by a slightly attractive contribution of the covalent interaction. A lower value of the dispersion interaction is found compared to the $\mathrm{Pt}_{37}$ systems
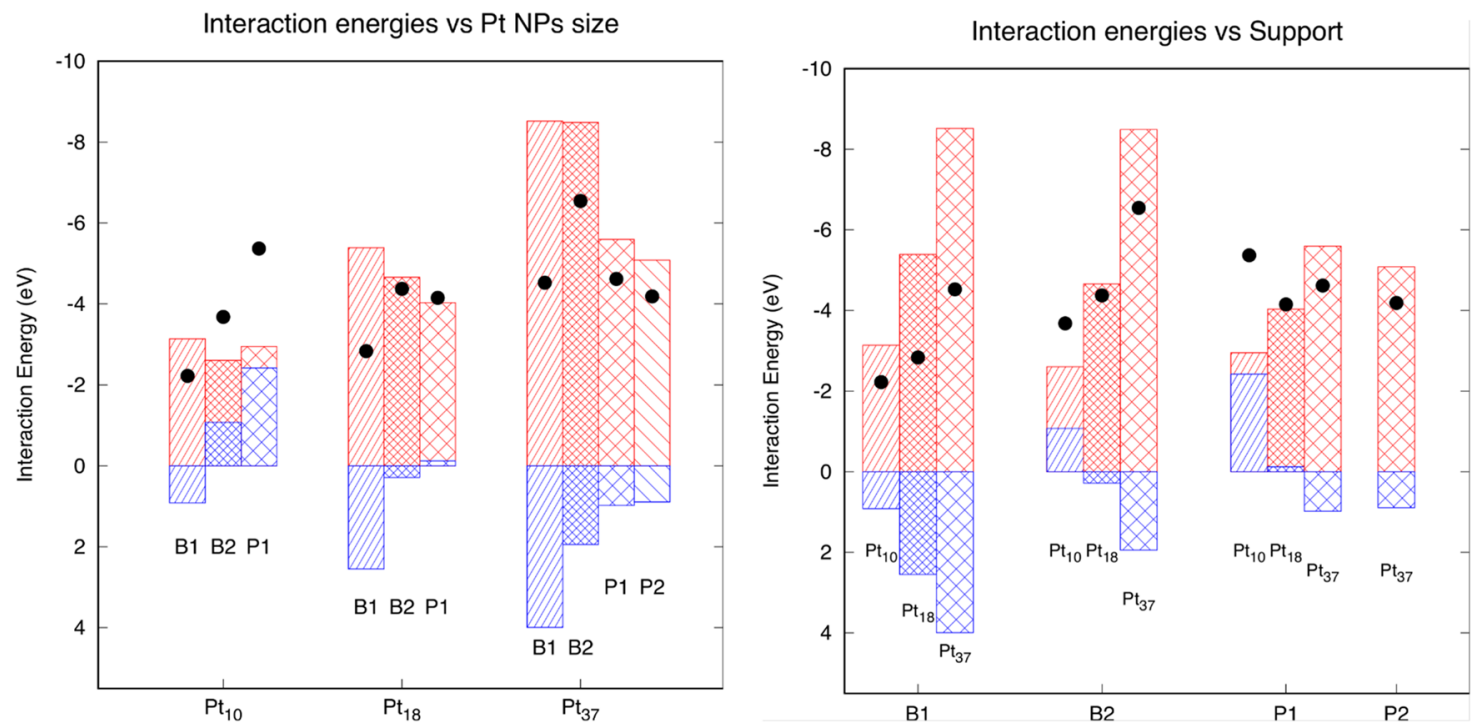

FIG. 7. Black dots: interaction energies between the Pt NPs and the different supports (B1 and B2 are the non-radical and radical basal planes and P1 and P2 are the planar and wavy prism faces); red boxes: dispersive contribution to the interaction energy; blue boxes: covalent contributions to the interaction energy. Right: interaction energies and their decompositions for each NP size as a function of the support. Left: interaction energies and their decompositions for each support as a function of as a function of the NP particle size. 

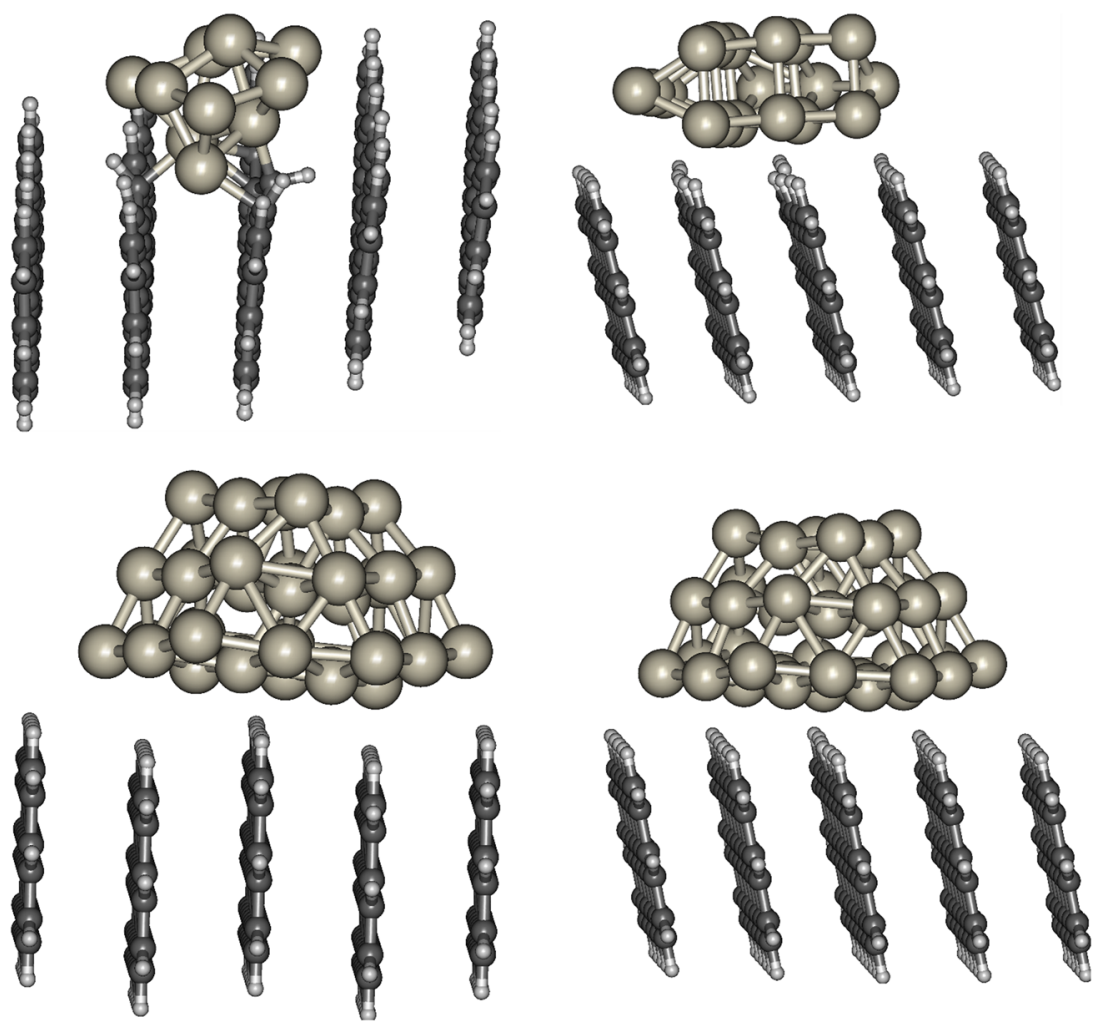

FIG. 8. Optimized structures of the different $\mathrm{Pt}$ nanoparticles at prism face models.

and can again be attributed to the decrease of the number of platinum atoms in the particle. Yet, the overall interaction energy is calculated to be close to the one of the $\mathrm{Pt}_{37}$ clusters. These phenomena are accentuated in the case of the $\mathrm{Pt}_{10}$ cluster as the NP penetrates deeper between the support layers (see Fig. 8). The minimum Pt-C distances are about $2.1 \AA$, which is comparable to what was obtained for the Pt particles at the zig-zag edges (B2). The covalent contribution to the interaction energy represents $44 \%$ of the total interaction energy. Even with the significant decrease of the dispersion interaction, due to fewer atoms that are involved, the total interaction energy between $\mathrm{Pt}_{10}$ and $\mathrm{P} 1$ is calculated to be the highest in the series. It is worth noting that in all cases, no cleavage of a $\mathrm{C}-\mathrm{H}$ bond is observed and that their lengths remain almost unchanged by the presence of the NPs.

\section{Charge transfer analysis}

Charge transfer between Pt nanoparticles and graphitic supports has been studied to understand structural changes in the nanoparticle upon interaction. For instance, RamosSanchez and Balbuena ${ }^{22}$ and Verga et al. ${ }^{19}$ reported a small charge transfer (about 0.1 electron) from the metallic cluster to the carbon support, an extended graphene sheet (periodic boundary conditions). In both studies, the charge transfer was calculated to slightly increase with the increase of the particle size. Contrary to these studies, in all our cases, a clear charge transfer was found from the carbon support to the
Pt NP (see Fig. 9), consistent with the fact that in all cases, the energy of the HOMO of the carbon support is higher than the electron chemical potential of the Pt nanoparticles

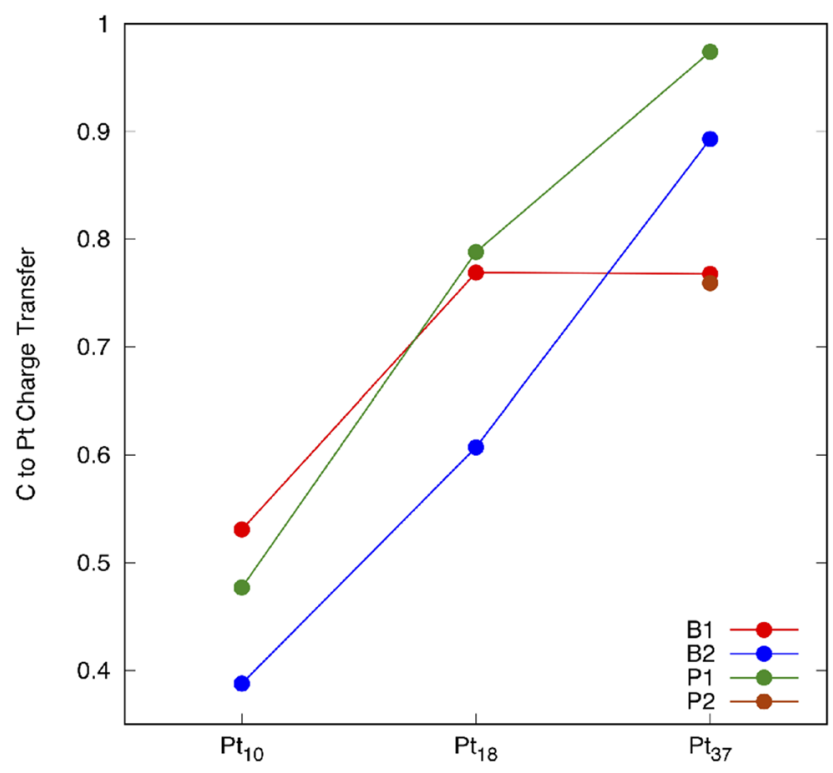

FIG. 9. Carbon support to Pt NP charge transfer for the different systems based on Hirshfeld charge analysis. 


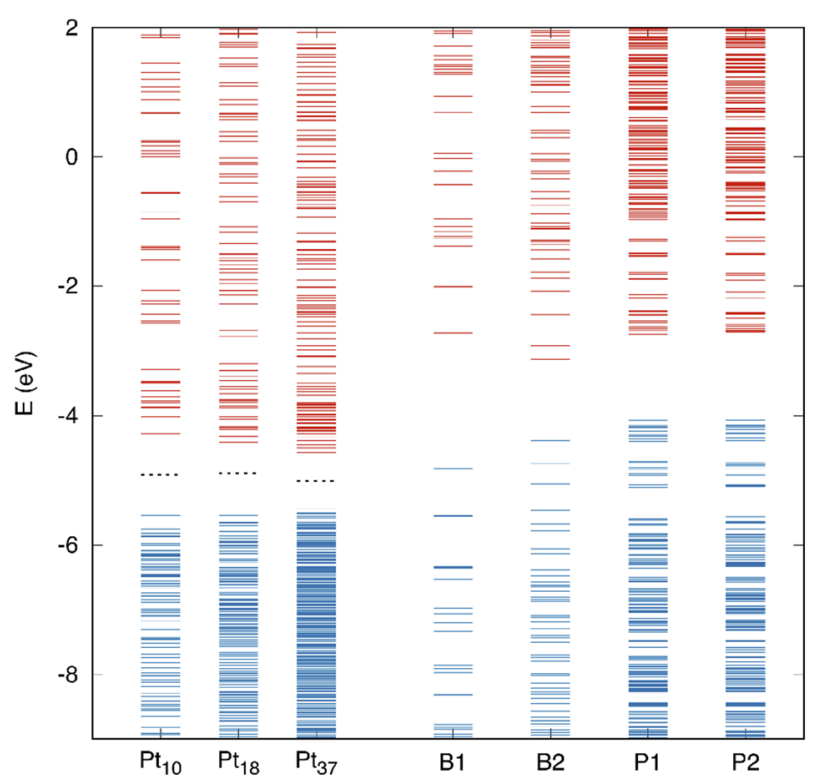

FIG. 10. Pt NPs and carbon supports occupied (blue) and virtual (red) molecular orbital energies and the electron chemical potential of the Pt NPs (black dotted lines) calculated at the RIJCOSX-B3LYP-D3/def2-TZVP level of theory.

(see Fig. 10). An exact estimate of the trend of the amount of charge transfer is far from being simple even when using the same method, such as Hirshfeld charge analysis, as the nature of the interaction and therefore $\mathrm{Pt}-\mathrm{C}$ distances vary from system to system. Thus, correlation between the size of Pt and C support with the charge transfer is hard to precisely extrapolate from this dataset. Nonetheless, our results suggest that the bigger the NP, the larger the charge transfer. Additionally, the charge transfer is mainly localized in the region between the Pt cluster and the carbon support, as shown for the Pt37 at the B1 system in Fig. S2 of the supplementary material and thus seems to have a limited influence on the edges of the support, which are anticipated to be more vulnerable to degradation.

\section{Influence of the potential on the charge state}

So far, our models were calculated in vacuum and no external potential was taken into account. However, as mentioned previously, most of the catalyst degradation occurs at a high potential, either at start-up and shut-down or under operating conditions of the oxygen reduction reaction (ORR), $1.23 \mathrm{~V}$ vs SHE. Thus, if one wants to gain insight into its mechanism, the influence of the potential has to be taken into account. By calculating the different redox potentials of the metal cluster, the carbon support, and the interacting system, one can have access to the domain of potential at which each of their charge states is thermodynamically stable. This has been done for the $\mathrm{Pt}_{37}$ cluster on the $\mathrm{B} 1$ model, and the results are shown in Fig. 11(a) for calculation in vacuum and Fig. 11(b) using a CPCM model of water at the geometry in the neutral state. Note that the electrochemical potential, or equally the Fermi level, of each species is exactly located at the center of each segment on the graph.

One can see that the carbon support is calculated to be stable as a neutral species between -2.5 and $1.2 \mathrm{~V}$ in vacuum. The neutral state of the $\mathrm{Pt}_{37}$ cluster is reduced and oxidized at higher potentials than the carbon support, -0.8 and $1.8 \mathrm{~V}$, respectively. The same trend can be observed for all calculated charge states. If we now focus on the high potential regime (above $0 \mathrm{~V}$ ) and consider the interacting system, the first oxidation potential is calculated to be even lower than that of the B1 support. As the two moieties are interacting, one cannot simply consider that the first oxidation can be viewed as removing an electron from the support. Indeed, as discussed earlier, already at the neutral state of the interacting system,

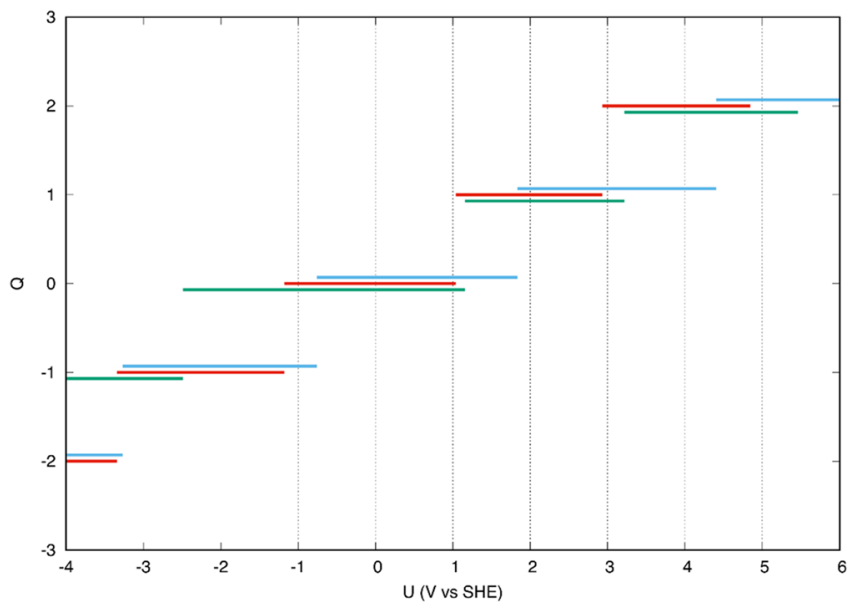

(a)

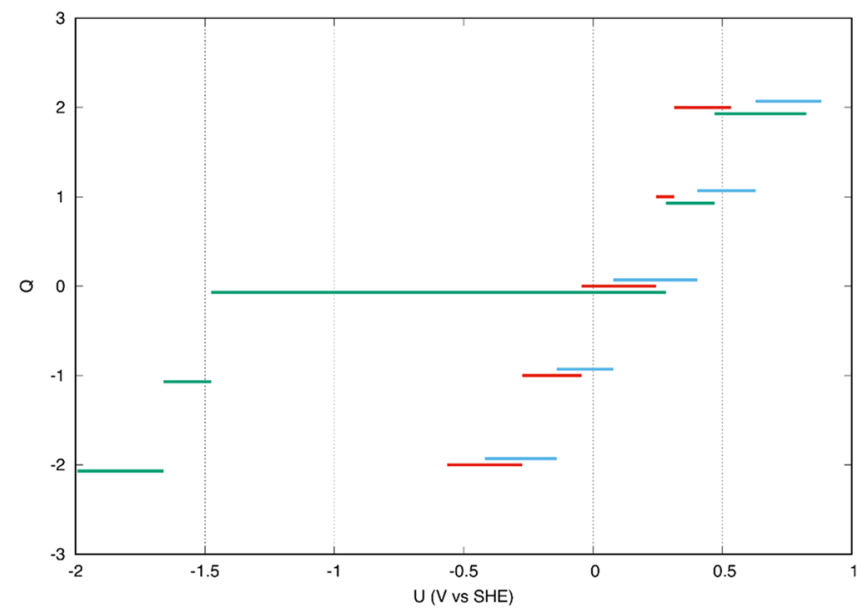

(b)

FIG. 11. Charge state, $Q$ (integer values), as a function of the potential, $\mathrm{U}$ (in V vs SHE), of $\mathrm{B} 1$ (green lines), $\mathrm{Pt}_{37}$ (blue lines), and $\mathrm{Pt}_{37} @ \mathrm{~B} 1$ (red lines) calculated at their optimum geometries in (a) vacuum and (b) using CPCM(water) at the D3-B3LYP/def2-TZVP level. 
TABLE III. Hirshfeld charges of the $\mathrm{B} 1$ support and the $\mathrm{Pt}_{37}$ cluster as a function of the charge at the optimized geometry of the $\mathrm{Q}=0$ charge state of $\mathrm{Pt}_{37} @ \mathrm{~B} 1$.

\begin{tabular}{lllllr}
\hline & \multicolumn{2}{c}{ Geometry Q $=0$} & & \multicolumn{2}{c}{$\begin{array}{c}\text { Optimized geometry } \\
\text { with less constraints }\end{array}$} \\
\cline { 2 - 3 } $\mathrm{Q}$ & $\mathrm{B} 1$ & $\mathrm{Pt}_{37}$ & & $\mathrm{~B} 1$ & $\mathrm{Pt}_{37}$ \\
\hline 2 & 1.90 & 0.10 & & 1.89 & 0.11 \\
1 & 1.13 & -0.13 & & 1.43 & -0.43 \\
0 & 0.77 & -0.77 & & 0.75 & -0.75 \\
-1 & 0.49 & -1.49 & & $\cdots$ & $\cdots$ \\
-2 & 0.20 & -2.20 & & $\cdots$ & $\cdots$ \\
\hline \hline
\end{tabular}

the B1 support is partially oxidized and consequently the Pt NP is partially reduced. Thus, upon oxidation of the neutral interacting system, about two thirds of the charge is removed from the NP (see Table III). On the other hand, in the second oxidation, about three fourths of the charge is removed from the support. Yet in all calculated charge states, the Pt NP is more negative than the carbon support.

When including the solvation model, the support, the NP, and the interacting system are oxidized at lower potentials, but the different trends observed in vacuum are rather conserved. The first oxidation of the carbon support is now at only $0.3 \mathrm{~V}$. Additionally, its charge is calculated to be larger than two at a potential of $1 \mathrm{~V}$. Note that it is likely that the solvation model overestimates the stabilization of the charged states compared to the actual environment in the experiment setup. However, the carbon support is likely to be oxidized in the range of the potential of the ORR and therefore deteriorates in the presence of $\mathrm{O}_{2}$ or $\mathrm{H}_{2} \mathrm{O}$. From a modeling point of view, it seems clear that in order to reach a more accurate description of the system under working conditions, several charge states have to be considered in order to account for the influence of the potential.

While this discussion relates to calculations done at fixed geometries (that of the neutral states), the last part of this work will investigate the effect of the charge state on the geometry and the nature of the interaction between the $\mathrm{Pt}_{37}$ NP and the B1 support.

\section{E. Influence of the potential on the interaction}

From the Hirshfeld charge analysis (Table III), one can see that the carbon support is almost mono-cationic at $\mathrm{Q}=1$ and di-cationic at $Q=2$ in the interacting system calculated at the geometry of the $\mathrm{Q}=0$ state. From the calculation of $\mathrm{B} 1$ at these two charge states, we know that their electronic ground states are open shell in both cases, doublet and open shell singlet, respectively. As for the B2 support, the radical(s) are located at the zig-zag edges of the PAH. So, to see if the same type of interaction as with the B2 support could be observed, this cluster was optimized at these two charge states. The thus obtained structures (Fig. 12) show a covalent type interaction with one of the edge carbon atom, and the minimum Pt-C distances are 2.3 and $2.2 \AA$, respectively, hence about the same as in the B2 case. While the presence of radical PAHs accessible to the NPs in experiment might be arguable because they might have already had reacted with $\mathrm{O}_{2}$ or $\mathrm{H}_{2} \mathrm{O}$ molecules, upon increase of the potential, an originally closed shell PAH can become an open shell. This will change the nature and the strength of the Pt NP support interaction.

\section{CONCLUSION}

State of the art HR TEM images allowed us to have access to atomically resolved features of Pt NPs on $\mathrm{CB}$ electrocatalysts. For the Pt nanoparticles, cuboctahedral or truncated-octahedral shaped particles were found with average sizes between 1.5 and $2.7 \mathrm{~nm}$. Additionally, the preference for the platinum (111) planes as the contact sites was confirmed. The carbon flakes (PAHs) constituting the CB material were found to be between 1 and a few nm large. The morphology of the contact area could be resolved and showed a congruent deformation of the Pt NPs and the PAHs in their vicinities. These data were used to construct relevant and computationally accessible models of both the Pt NPs and the carbon support.

Our computational study confirmed that the interaction of Pt NPs and graphene basal plane supports is dominated by dispersion interaction which is also true, yet to a lesser extent, for the graphite prism face (Fig. 13). The radical character of PAH zig-zag edges induces covalent bonds, even for the large NP, between Pt atoms and carbon edge atoms. The structure thus obtained shows similar deformations of both the NP and the carbon support as those observed on the HR TEM images. Additionally, due to the combination of dispersion and covalent interactions, Pt NPs at the basal plane with radical zig-zag edges are found to have the largest interaction energy. Hence the distortion from the well-shaped faces of the NPs observed on the HR TEM images can be rationalized based on electronic structure calculations. The distance between the $\mathrm{Pt}$ atoms at the interface and the carbon support is rather difficult to access from HR TEM images. However, from our computational results, we find Pt-C distances of $\sim 2.9 \AA$ for pure dispersion interaction, which is smaller than the $3.45 \AA$
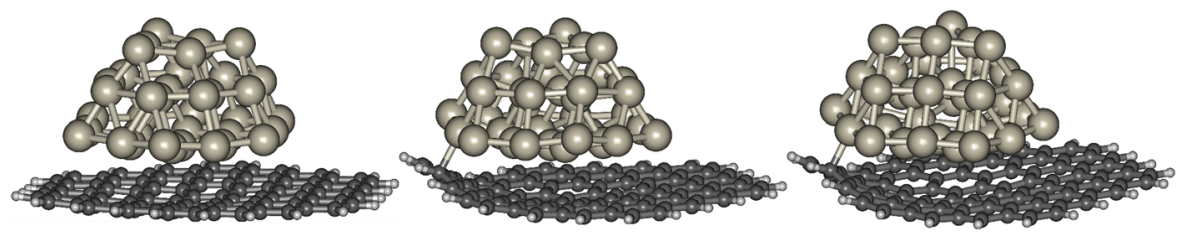

FIG. 12. Optimized structure of $\mathrm{Pt}_{37} @ \mathrm{~B} 1$, with constraints on the carbon support as depicted in Fig. 4, at charge states $Q=0$ (left), $Q=1$ (middle), and $Q=2$ (right). 


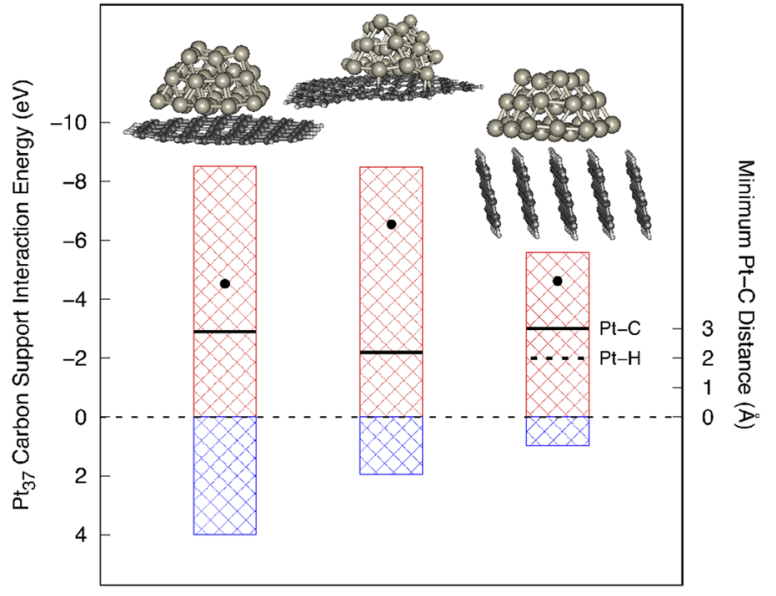

FIG. 13. Black dots: interaction energies between the Pt NPs and the different supports (B1 and B2 are the non-radical and radical basal planes and $\mathrm{P} 1$ is the planar prism faces); red boxes: dispersive contribution to the interaction energy; blue boxes: covalent contributions to the interaction energy. Horizontal solid and dashed black lines correspond to the minimum $\mathrm{Pt}-\mathrm{C}$ and $\mathrm{Pt}-\mathrm{H}$ distances (right y-axis), respectively.

corresponding to the sum of the two van der Waals radii, and of $\sim 2.2 \AA$ for covalent interaction.

Contrary to previous studies, we find a clear charge transfer from the carbon support to the Pt NPs in all cases, thus resulting in a partial oxidation of the support PAH(s). Furthermore, our study on the influence on the potential shows that upon increase of the potential, the carbon support is oxidized sooner than the Pt cluster and that the interacting system has a lower reduction potential that the PAH alone. Thus, the carbon support is found to be likely oxidized in the range of the potential of the ORR and therefore to be deteriorated in the presence of $\mathrm{O}_{2}$ or $\mathrm{H}_{2} \mathrm{O}$. Additionally, it was found that the potential can also change the nature of the interaction between a Pt NP and a PAH. Indeed, the structures obtained for charge states corresponding to potentials larger than 0 $\mathrm{V}$ show an increase of the covalent character of the Pt NP carbon support interaction through shorter $\mathrm{Pt}-\mathrm{C}$ distances at the PAH edge. Thus, the influence of the potential cannot be neglected when modeling electrocatalytic systems, especially in the rather molecular framework of NPs at assembled PAHs, as one might miss some specific features not present in the models' neutral state.

\section{SUPPLEMENTARY MATERIAL}

See supplementary material for a figure on combustion energies per carbon for the carbon supports, a detailed table on Pt@Support interaction (energies, decomposition, and charge transfer values), the charge density difference plot of $\mathrm{Pt}_{37} @ \mathrm{~B} 1$, general Orca input used in this work, and the optimized structures of all the systems presented in this study.

\section{ACKNOWLEDGMENTS}

The authors would like to acknowledge funding by the BMWi (Project No. PtTM@HGS FKZ 03ET6080C). Alexander Auer would like to acknowledge additional funding by the BMBF (Project No. JointLab GEP FKZ 13XP5023E), the Max-Planck Society, and the Max-Planck research initiative MAXNET ENERGY, and C. Freysoldt (MPIE) for helpful discussions. The authors are grateful to Dr. Juri Barthel for the support with the PICO microscope.

\section{REFERENCES}

${ }^{1}$ S. Guo, S. Zhang, and S. Sun, "Tuning nanoparticle catalysis for the oxygen reduction reaction," Angew. Chem., Int. Ed. 52, 8526-8544 (2013).

${ }^{2}$ P. Mani, R. Srivastava, and P. Strasser, "Dealloyed Pt-Cu core-shell nanoparticle electrocatalysts for use in PEM fuel cell cathodes," J. Phys. Chem. C 112, 2770-2778 (2008).

${ }^{3}$ J. Chen, B. Lim, E. P. Lee, and Y. Xia, "Shape-controlled synthesis of platinum nanocrystals for catalytic and electrocatalytic applications," Nano Today 4, 81-95 (2009)

${ }^{4}$ C. Wang, H. Daimon, T. Onodera, T. Koda, and S. Sun, "A general approach to the size- and shape-controlled synthesis of platinum nanoparticles and their catalytic reduction of oxygen," Angew. Chem., Int. Ed. 47, 3588-3591 (2008).

${ }^{5}$ S. Sharma and B. G. Pollet, "Support materials for PEMFC and DMFC electrocatalysts-A review," J. Power Sources 208, 96-119 (2012).

${ }^{6} \mathrm{~J}$. C. Calderón, L. Ndzuzo, B. J. Bladergroen, and S. Pasupathi, "Catalytic activity of carbon supported-Pt-Pd nanoparticles toward the oxygen reduction reaction," Mater. Today: Proc. 5, 10551-10560 (2018).

${ }^{7}$ F. Montilla, E. Morallón, I. Duo, C. Comninellis, and J. L. Vázquez, "Platinum particles deposited on synthetic boron-doped diamond surfaces. Application to methanol oxidation," Electrochim. Acta 48, 3891-3897 (2003).

${ }^{8} \mathrm{Q}$. Ren, H. Wang, X. Lu, Y. Tong, and G. Li, "Recent progress on MOF-derived heteroatom-doped carbon-based electrocatalysts for oxygen reduction reaction," Adv. Sci. 5, 1700515 (2018).

${ }^{9}$ Y. L. Hsin, K. C. Hwang, and C.-T. Yeh, "Poly(vinylpyrrolidone)-modified graphite carbon nanofibers as promising supports for PtRu catalysts in direct methanol fuel cells," J. Am. Chem. Soc. 129, 9999-10010 (2007).

${ }^{10}$ E. Antolini, "Carbon supports for low-temperature fuel cell catalysts," Appl. Catal., B 88, 1-24 (2009).

${ }^{11}$ C. Zhang, X. Shen, Y. Pan, and Z. Peng, "A review of Pt-based electrocatalysts for oxygen reduction reaction," Front. Energy 11, 268-285 (2017).

${ }^{12}$ C. Hartnig and T. J. Schmidt, "Simulated start-stop as a rapid aging tool for polymer electrolyte fuel cell electrodes," J. Power Sources 196, 5564-5572 (2011).

${ }^{13} \mathrm{~J}$. C. Meier et al., "Degradation mechanisms of $\mathrm{Pt} / \mathrm{C}$ fuel cell catalysts under simulated start-stop conditions," ACS Catal. 2, 832-843 (2012).

${ }^{14}$ Y. Shao, G. Yin, and Y. Gao, "Understanding and approaches for the durability issues of Pt-based catalysts for PEM fuel cell," J. Power Sources 171, 558-566 (2007).

${ }^{15} \mathrm{G}$. Bae, D. H. Youn, S. Han, and J. S. Lee, "The role of nitrogen in a carbon support on the increased activity and stability of a Pt catalyst in electrochemical hydrogen oxidation," Carbon 51, 274-281 (2013).

${ }^{16} \mathrm{C}$. Galeano et al., "Toward highly stable electrocatalysts via nanoparticle pore confinement," J. Am. Chem. Soc. 134, 20457-20465 (2012).

${ }^{17} \mathrm{~L}$. Xiao and L. Wang, "Structures of platinum clusters: Planar or spherical?," J. Phys. Chem. A 108, 8605-8614 (2004). 
${ }^{18} \mathrm{~K}$. Okazaki-Maeda, Y. Morikawa, S. Tanaka, and M. Kohyama, "Structures of Pt clusters on graphene by first-principles calculations," Surf. Sci. 604, 144-154 (2010).

${ }^{19}$ L. G. Verga et al., "Effect of graphene support on large Pt nanoparticles," Phys. Chem. Chem. Phys. 18, 32713-32722 (2016).

${ }^{20} \mathrm{P}$. Błoński and J. Hafner, "Geometric and magnetic properties of Pt clusters supported on graphene: Relativistic density-functional calculations," J. Chem. Phys. 134, 154705 (2011).

${ }^{21}$ W. B. Schneider, U. Benedikt, and A. A. Auer, "Interaction of platinum nanoparticles with graphitic carbon structures: A computational study," ChemPhysChem 14, 2984-2989 (2013).

${ }^{22} \mathrm{G}$. Ramos-Sanchez and P. B. Balbuena, "Interactions of platinum clusters with a graphite substrate," Phys. Chem. Chem. Phys. 15, 11950-11959 (2013).

${ }^{23} \mathrm{~A}$. Maiti and A. Ricca, "Metal-nanotube interactions-binding energies and wetting properties," Chem. Phys. Lett. 395, 7-11 (2004).

${ }^{24}$ C. F. Sanz-Navarro et al., "Molecular dynamics simulations of the interactions between platinum clusters and carbon platelets," J. Phys. Chem. A 112, 1392-1402 (2008).

${ }^{25}$ B. H. Morrow and A. Striolo, "Platinum nanoparticles on carbonaceous materials: The effect of support geometry on nanoparticle mobility, morphology, and melting," Nanotechnology 19, 195711 (2008).

${ }^{26} \mathrm{I}$. Fampiou and A. Ramasubramaniam, "Binding of Pt nanoclusters to point defects in graphene: Adsorption, morphology, and electronic structure," J. Phys. Chem. C 116, 6543-6555 (2012).

${ }^{27} \mathrm{Y}$. Okamoto, "Density-functional calculations of icosahedral $\mathrm{M}_{13}(\mathrm{M}=\mathrm{Pt}$ and $\mathrm{Au}$ ) clusters on graphene sheets and flakes," Chem. Phys. Lett. 420, 382-386 (2006).

${ }^{28}$ H.-H. Liu, K.-L. Hsueh, and C.-W. Hong, "DFT analysis on the Pt with nano-carbon frames for low temperature fuel cell applications," Electrochim. Acta 259, 598-605 (2018).

${ }^{29}$ D.-H. Lim and J. Wilcox, "DFT-based study on oxygen adsorption on defective graphene-supported Pt nanoparticles," J. Phys. Chem. C 115, 22742-22747 (2011).

${ }^{30} \mathrm{~S}$. Grimme, "Semiempirical GGA-type density functional constructed with a long-range dispersion correction," J. Comput. Chem. 27, 1787-1799 (2006).

${ }^{31}$ S. Grimme, J. Antony, S. Ehrlich, and H. Krieg, "A consistent and accurate $a b$ initio parametrization of density functional dispersion correction (DFTD) for the 94 elements H-Pu," J. Chem. Phys. 132, 154104 (2010).

${ }^{32}$ P. Paciok, M. Schalenbach, M. Carmo, and D. Stolten, "On the mobility of carbon-supported platinum nanoparticles towards unveiling cathode degradation in water electrolysis," J. Power Sources 365, 53-60 (2017).

${ }^{33} \mathrm{M}$. S. Moldovan et al., "On the evolution of Pt nanoparticles on few-layer graphene supports in the high-temperature range," J. Phys. Chem. C 116, 9274-9282 (2012).

${ }^{34} \mathrm{P}$. Trogadas, T. F. Fuller, and P. Strasser, "Carbon as catalyst and support for electrochemical energy conversion," Carbon 75, 5-42 (2014).

${ }^{35} \mathrm{Z}$. Sun, Z. Zeng, and J. Wu, "Zethrenes, extended p-quinodimethanes, and periacenes with a singlet biradical ground state," Acc. Chem. Res. 47, 25822591 (2014).

${ }^{36} \mathrm{Z}$. Sun et al., "Dibenzoheptazethrene isomers with different biradical characters: An exercise of clar's aromatic sextet rule in singlet biradicaloids," J. Am. Chem. Soc. 135, 18229-18236 (2013).

${ }^{37} \mathrm{Z}$. Sun and J. Wu, "Open-shell polycyclic aromatic hydrocarbons," J. Mater. Chem. 22, 4151-4160 (2012)

${ }^{38}$ F. Plasser, H. Pašalić, M. H. Gerzabek, F. Libisch, R. Reiter, J. Burgdörfer, T. Müller, R. Shepard, and H. Lischka, "The multiradical character of oneand two-dimensional graphene nanoribbons," Angew. Chem., Int. Ed. 52, 2581-2584 (2013)
${ }^{39}$ A. V. Luzanov, F. Plasser, A. Das, and H. Lischka, "Evaluation of the quasi correlated tight-binding (QCTB) model for describing polyradical character in polycyclic hydrocarbons," J. Chem. Phys. 146, 064106 (2017).

${ }^{40}$ Y. Ruiz-Morales, "The agreement between clar structures and nucleusindependent chemical shift values in pericondensed benzenoid polycyclic aromatic hydrocarbons: An application of the Y-rule," J. Phys. Chem. A 108 , 10873-10896 (2004).

${ }^{41}$ A. Shimizu et al., "Theoretical consideration of singlet open-shell character of polyperiacenes using Clar's aromatic sextet valence bond model and quantum chemical calculations," AIP Conf. Proc. 1504, 399-405 (2012).

${ }^{42}$ G. Trinquier, N. Suaud, and J.-P. Malrieu, "Theoretical design of high-spin polycyclic hydrocarbons," Chem. - Eur. J. 16, 8762-8772 (2010).

${ }^{43}$ G. Trinquier and J.-P. Malrieu, "Kekulé versus Lewis: When aromaticity prevents electron pairing and imposes polyradical character," Chem. Eur. J. 21, 814-828 (2015)

${ }^{44} \mathrm{~A}$. Konishi et al., "Synthesis and characterization of quarteranthene: Elucidating the characteristics of the edge state of graphene nanoribbons at the molecular level," J. Am. Chem. Soc. 135, 1430-1437 (2013).

${ }^{45}$ W. B. Schneider and A. A. Auer, "Constant chemical potential approach for quantum chemical calculations in electrocatalysis," Beilstein J. Nanotechnol. 5, 668-676 (2014).

${ }^{46}$ U. Benedikt, W. B. Schneider, and A. A. Auer, "Modelling electrified interfaces in quantum chemistry: Constant charge vs. constant potential," Phys. Chem. Chem. Phys. 15, 2712 (2013)

${ }^{47}$ F. Neese, "The ORCA program system," Wiley Interdiscip. Rev.: Comput. Mol. Sci. 2, 73-78 (2012).

${ }^{48}$ A. D. Becke, "Density-functional thermochemistry. III. The role of exact exchange," J. Chem. Phys. 98, 5648-5652 (1993)

${ }^{49}$ C. Lee, W. Yang, and R. G. Parr, Phys. Rev. B 37, 785 (1988).

${ }^{50} \mathrm{~F}$. Neese, F. Wennmohs, A. Hansen, and U. Becker, "Efficient, approximate and parallel Hartree-Fock and hybrid DFT calculations. A 'chain-of-spheres' algorithm for the Hartree-Fock exchange," Chem. Phys. 356, 98-109 (2009).

${ }^{51} \mathrm{~S}$. Grimme, S. Ehrlich, and L. Goerigk, "Effect of the damping function in dispersion corrected density functional theory," J. Comput. Chem. 32, 14561465 (2011)

${ }^{52} \mathrm{~F}$. Weigend and R. Ahlrichs, "Balanced basis sets of split valence, triple zeta valence and quadruple zeta valence quality for $\mathrm{H}$ to $\mathrm{Rn}$ : Design and assessment of accuracy," Phys. Chem. Chem. Phys. 7, 3297-3305 (2005).

${ }^{53} \mathrm{~F}$. Weigend, "Accurate Coulomb-fitting basis sets for $\mathrm{H}$ to Rn," Phys. Chem. Chem. Phys. 8, 1057-1065 (2006).

${ }^{54}$ D. Andrae, U. Häußermann, M. Dolg, H. Stoll, and H. Preuß, "Energyadjustedab initio pseudopotentials for the second and third row transition elements," Theor. Chim. Acta 77, 123-141 (1990).

${ }^{55} \mathrm{~S}$. Trasatti, "The absolute electrode potential: An explanatory note (recommendations 1986)," Pure Appl. Chem. 58, 955-966 (2009).

${ }^{56}$ C. L. Jia, L. Houben, A. Thust, and J. Barthel, "On the benefit of the negative-spherical-aberration imaging technique for quantitative HRTEM," Ultramicroscopy 110, 500-505 (2010).

${ }^{57} \mathrm{E}$. Willinger et al., "Characterization of the platinum-carbon interface for electrochemical applications," ACS Catal. 7, 4395-4407 (2017).

${ }^{58}$ J. E. Anthony, "The larger acenes: Versatile organic semiconductors," Angew. Chem., Int. Ed. 47, 452-483 (2008).

${ }^{59} \mathrm{M}$. Head-Gordon, "Characterizing unpaired electrons from the oneparticle density matrix," Chem. Phys. Lett. 372, 508-511 (2003). 